\title{
Influence of Nitrogen Vacancy Concentration on Mechanical and Electrical Properties of Rocksalt Zirconium Nitride Films
}

\author{
Ke-Chang Han ${ }^{1}$ Guo-Qiang Lin ${ }^{1} \cdot$ Chuang Dong ${ }^{1} \cdot$ Kai-Ping Tai $^{2} \cdot$ Xin Jiang $^{2}$
}

Received: 9 May 2017/Revised: 30 June 2017/Published online: 18 August 2017

(C) The Chinese Society for Metals and Springer-Verlag GmbH Germany 2017

\begin{abstract}
To study the influence of the nitrogen vacancy $\left(V_{\mathrm{N}}\right)$ on mechanical and electrical properties of zirconium nitride deeply, $\mathrm{ZrN}_{x}$ films with different $V_{\mathrm{N}}$ concentrations were synthesized on the $\mathrm{Si}$ (111) substrates by enhanced magnetic filtering arc ion plating. The morphologies, microstructures, residual stresses, compositions, chemical states, mechanical and electrical properties of the as-deposited films were characterized by field-emission scanning electron microscopy, $\mathrm{X}$-ray diffraction, X-ray photoelectron spectrometry, Nanoindenter and Hall effect measurements. The results showed that $\mathrm{ZrN}_{x}$ films exhibited rocksalt single-phase structure within a $V_{\mathrm{N}}$ concentration ranging from 26 to 5\%. The preferred orientation, thickness, grain size and residual stress of the $\mathrm{ZrN}_{x}$ films kept constant at different $V_{\mathrm{N}}$ concentrations. Both the nanohardness and elastic modulus first increased and then decreased with the decrease in $V_{\mathrm{N}}$ concentration, reaching the peaks around $16 \%$. And the electric conductivity of the $\mathrm{ZrN}_{x}$ films showed a similar tendency with nanohardness. The underlying atomic-scale mechanisms of $V_{\mathrm{N}}$ concentration-dependent hardness and electric conductivity enhancements were discussed and attributed to the different electronic band structures, rather than conventional meso-scale factors, such as preferred orientation, grain size and residual stress.
\end{abstract}

KEY WORDS: $\mathrm{ZrN}_{x}$ films; Nitrogen vacancy; Hardness; Electrical properties; Band structure

\section{Introduction}

Transition-metal nitrides (TMNs) are well known as a class of fascinating and technologically important ceramic materials which crystallize normally in the rocksalt (B1) structure [1,2]. The close-packed rocksalt structure hinders the migration of species, with the result of excellent thermal and chemical stability against, for example, oxidation

Available online at http://link.springer.com/journal/40195

Guo-Qiang Lin

gqlin@dlut.edu.cn

1 Key Laboratory for Material Modification by Laser, Ion and Electron Beams, (Ministry of Education), Dalian University of Technology, Dalian 116024, China

2 Institute of Metal Research, Chinese Academy of Sciences, Shenyang 110016, China or self-diffusion [3]. As a typical one of TMNs, zirconium nitride $(\mathrm{ZrN})$ has been widely used as a coating material, ranging from hard and protective coatings on mechanical tools, wear resistant and decorative layers on optical components, to the diffusion barriers in microelectronics owing to its exceptional thermal and mechanical properties, and electrical conductivity [4-6]. And intriguingly, $\mathrm{ZrN}_{x}$ has the wide solid solution region, i.e., retains the rocksalt structure within large composition range [7, 8]. This provides the possibility to tailor its material properties by selecting specific stoichiometric ratio.

Nitrogen vacancy $\left(V_{\mathrm{N}}\right)$ normally exists in substoichiometric $\mathrm{ZrN}_{x}$ phases and is regarded as the primary defect that controls the stoichiometric ratio [7]. Therefore, $V_{\mathrm{N}}$ engineering has been the focus of some experimental studies attempting to tailor the properties of $\mathrm{ZrN}_{x}$. Several studies have revealed that its mechanical properties strongly depend on the $V_{\mathrm{N}}$ concentration and the tendency 
does not follow the monotonous rule that vacancies reduce the number of chemical bonds and hence the strength of materials [9-11]. For instance, Abdallah et al. [10] reported that the maximal hardness was obtained at $V_{\mathrm{N}}$ concentration of $9 \%$ and Huang et al. [11] got the maximal hardness at $V_{\mathrm{N}}$ concentration of $20 \%$. Similarly, the resistivity of $\mathrm{ZrN}_{x}$ film was also reported to reach its bottom at the specific substoichiometric point such as $V_{\mathrm{N}}$ concentration of $21 \%$ by Khan et al. [12]. However, the unusual mechanical and electrical properties behaviors were often attributed to the influence of conventional microstructural features, such as preferred orientation, residual stress and grain size [10-12]. The underlying atomic-scale mechanisms and pathways leading to the development of mechanical and electrical properties are still concealed by the conventional meso-scale factors and not properly understood.

In this work, more delicate experiments were carried out to further study the influence of $V_{\mathrm{N}}$ concentration on mechanical and electrical properties of the rocksalt $\mathrm{ZrN}_{x}$ films and explore the underlying atomic-scale mechanisms. The enhanced magnetic filtering arc ion plating (MFAIP) was used to synthesize high-quality $\mathrm{ZrN}_{x}$ films with different $V_{\mathrm{N}}$ concentrations by varying the nitrogen flow rate. And other deposition parameters were precisely controlled to weaken the influence of conventional meso-scale factors as possible.

\section{Experimental}

$\mathrm{ZrN}_{x}$ films were deposited on the $\mathrm{Si}$ (111) substrates with a size of $15 \mathrm{~mm} \times 15 \mathrm{~mm}$ by the enhanced MFAIP system. Figure 1 shows the schematic diagram of the enhanced
MFAIP system. Two high-purity (99.99\%) Zr targets with a size of $55 \mathrm{~mm}$ in diameter were positioned symmetrically on two opposite sides of the chamber. A rotating sample holder was set in the center of the chamber. The straight magnetic filtering system consisted of two grade magnetic coils was placed before the targets and used to stream the plasma to the chamber utilizing plasma guiding by magnetic fields.

Before loading into the deposition chamber, the $\mathrm{Si}$ (111) substrates were cleaned in acetone and ethanol for $10 \mathrm{~min}$, respectively. When the base pressure in the chamber was down to $5.0 \times 10^{-3} \mathrm{~Pa}$, the substrates were preheated and then maintained at $200{ }^{\circ} \mathrm{C}$. Ar plasma at $1.6 \mathrm{~Pa}$ pressure was used to clean the surface of the substrates with a pulsed bias voltage of $850 \mathrm{~V} \times 30 \mathrm{kHz} \times 50 \%$ for $10 \mathrm{~min}$. During the deposition process, argon and nitrogen gases were fed into the chamber by mass flow controller and total working pressure was kept constant at $0.6 \mathrm{~Pa}$. The target currents were maintained at $90 \mathrm{~A}$, and the pulsed bias voltage was down to $300 \mathrm{~V} \times 30 \mathrm{kHz} \times 30 \%$. The total deposition time was 80 min. Seven groups of $\mathrm{ZrN}_{x}$ films with different $V_{\mathrm{N}}$ concentrations (S1-S7) were prepared by only varying the nitrogen flow rate from 60 to $270 \mathrm{sccm}$. Detailed nitrogen flow rates and percent of nitrogen in the plasma of $\mathrm{ZrN}_{x}$ films are listed in Table 1.

The field-emission scanning electron microscope (Zeiss Supera55) was utilized to observe the surface and crosssectional morphologies. The Bragg-Brentano X-ray diffraction (Bruker D8) was utilized to study the crystallographic structure of the $\mathrm{ZrN}_{x}$ films by using $\mathrm{Cu} K_{\alpha}$ radiation (wavelength $\lambda=0.154 \mathrm{~nm}$ ). Chemical states and compositions of the as-deposited films were examined by the X-ray photoelectron spectrometer (VG ESCALAB 250) with a monochromatic Al $K_{\alpha}$ source $(1486.6 \mathrm{eV})$. The

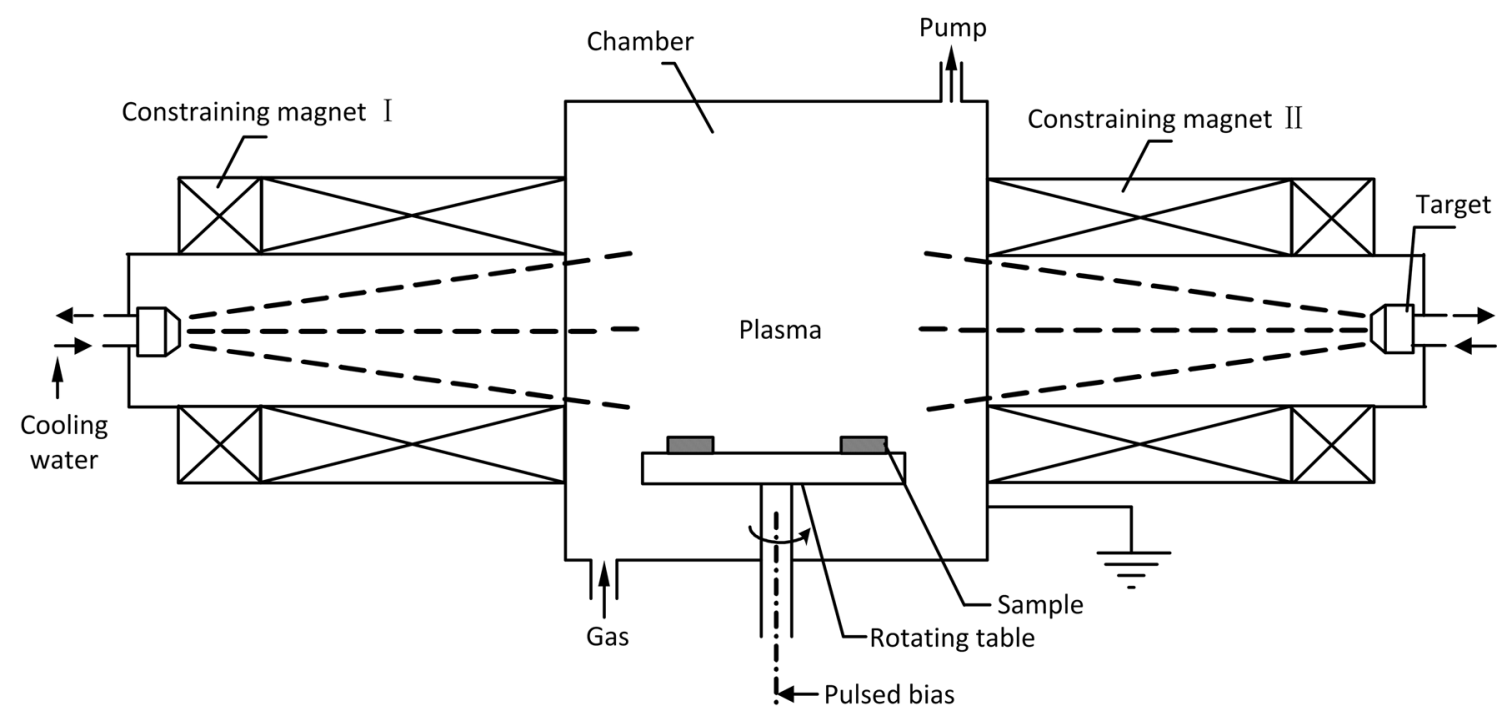

Fig. 1 Schematic diagram of enhanced magnetic filtering arc ion plating system 
Table 1 Nitrogen flow rate of $\mathrm{ZrN}_{x}$ films deposited by arc ion plating

\begin{tabular}{lrrrrrrr}
\hline Sample & S1 & S2 & S3 & S4 & S5 & S6 & S7 \\
\hline Nitrogen flow rate (sccm) & 60 & 120 & 150 & 180 & 210 & 240 & 270 \\
Percent of nitrogen in plasma (\%) & 16 & 30 & 37 & 43 & 49 & 55 & 60 \\
\hline
\end{tabular}

residual stress was determined by modified XRD $\sin ^{2} \psi(\psi$ is the angle between stress direction and surface normal direction) method using a four-circle diffractometer with psi-goniometer geometry [13]. Nanoindentation tests were carried out on a nano-indenter system (MTS XP) fitting with a Berkovich indenter under the continuous stiffness measurement mode, and the values were evaluated at about $10 \%$ of the film thickness to avoid the substrate effect. The electrical conductivity, carrier concentration and mobility were determined by Hall effect measurement system (Ecopia HMS-5000) at room temperature.

\section{Results}

\subsection{Surface Morphologies}

The typical surface and cross-sectional SEM images of $\mathrm{ZrN}_{x}$ films at different nitrogen flow rates are shown in Fig. 2. The surface is uniform, dense and fewer macroparticles are observed compared with traditional DC bias arc ion plating [14, 15]. The reasons for the improvement of film deposition quality mainly come from the adoption of enhanced magnetic filtering system and pulsed bias. The enhanced magnetic filtering system with two grades magnetic coils can effectively restrain the macroparticles from reaching the substrates. And the introduction of pulsed bias can form the oscillating plasma sheath above the substrates and induce more negative charging in the macroparticles and then improve the films deposition quality ulteriorly by coulomb repulsive effect between negatively charged macroparticles and substrate $[15,16]$. The thicknesses of $\mathrm{ZrN}_{x}$ films were determined by the cross-sectional images and found to be stable at around $1.17 \mu \mathrm{m}$ with the increase in nitrogen flow rate.

\subsection{Crystal Structure}

X-ray diffraction patterns of the $\mathrm{ZrN}_{x}$ films deposited at different nitrogen flow rates are presented in Fig. 3. The
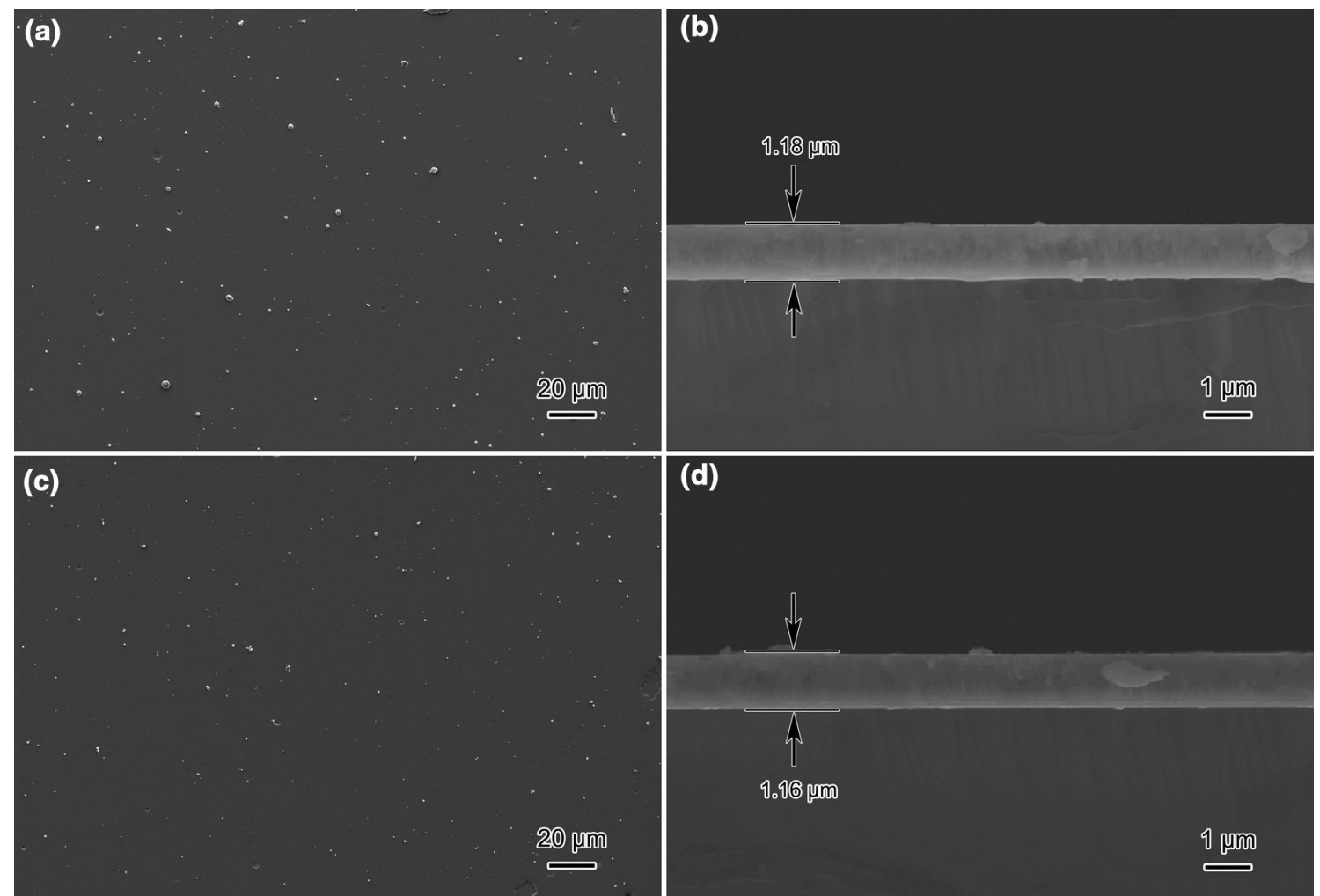

Fig. 2 Surface a, c and cross section b, d SEM images of $\mathrm{ZrN}_{x}$ films deposited at nitrogen flow rates of $60 \mathrm{sccm} \mathbf{a}, \mathbf{b}$ and $270 \mathrm{sccm} \mathbf{c}, \mathbf{d}$ 


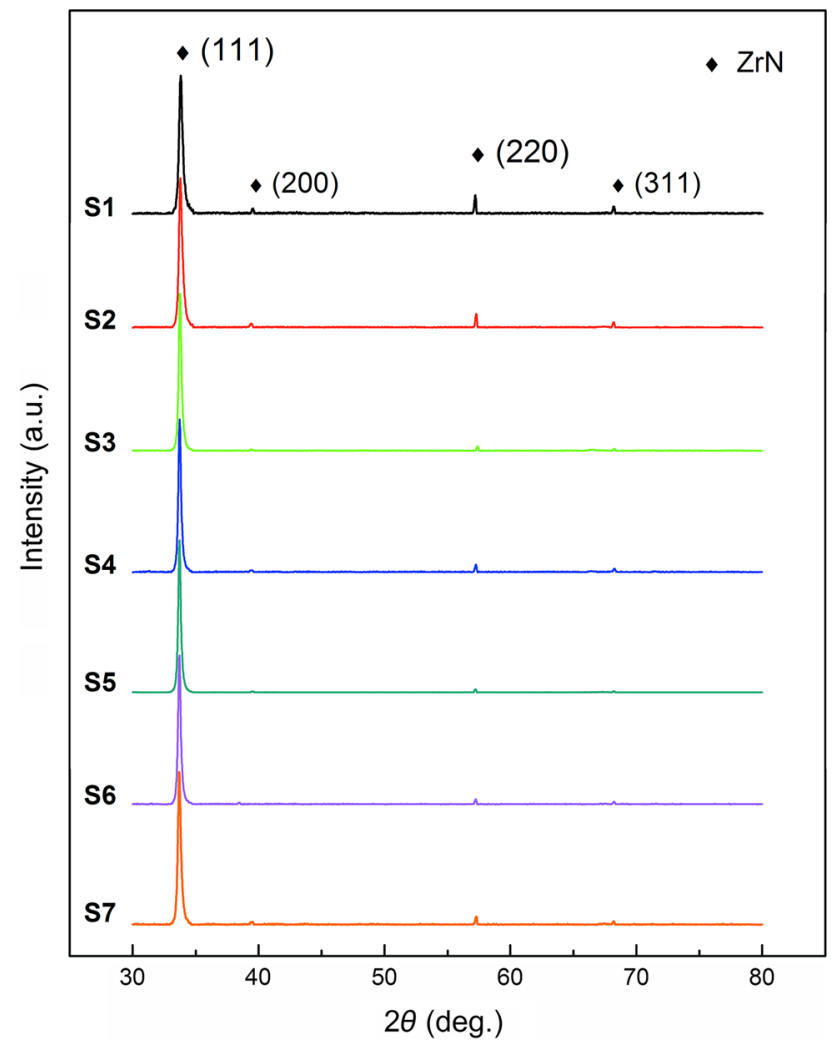

Fig. 3 XRD patterns of $\mathrm{ZrN}_{x}$ films deposited at different nitrogen flow rates

strongest peak corresponds to (111) crystal plane of the rocksalt structure $\mathrm{ZrN}$ (JCPDS\#35-0753). Other weak peaks corresponding to diffractions from $\mathrm{ZrN}$ (220), $\mathrm{ZrN}$ (200) and $\mathrm{ZrN}$ (311) are also observed. The patterns indicate that all the $\mathrm{ZrN}_{x}$ films perform the stable rocksalt structure and show the preferential growth along (111) orientation.

Figure 4a shows the lattice parameters of $\mathrm{ZrN}_{x}$ films deposited at different nitrogen flow rates. It can be seen that the lattice parameter increases monotonously with the increase in nitrogen flow rate. This may be attributed to the effect of $V_{\mathrm{N}}$ in the substoichiometric $\mathrm{ZrN}_{x}$ films. For the $\mathrm{TMN}_{x}$ films deposited at low nitrogen flow rate, $V_{\mathrm{N}}$ is believed to be formed in the crystal lattice as mentioned before, leading to the shrinkage of lattice parameter $[7,17,18]$. As the nitride flow rate increases, $V_{\mathrm{N}}$ concentration decreases and the shrinkage develops. And the rocksalt structure can be preserved maybe benefit from the suppression of migration and clustering of $V_{\mathrm{N}}$ by the large $V_{\mathrm{N}}$ diffusion barrier [7].

The position of the $\mathrm{ZrN}$ (111) diffraction peak and the corrected full width at half maximum (FWHM) are used to estimate the grain size by the Scherrer equation. The grain size changes within a small range from 30.0 to $31.4 \mathrm{~nm}$, maintaining nearly constant within the error ranges as shown in Fig. 4b. The grain size of thin film was reported to be controlled by the mobility of adatoms during growth, which were mainly determined by incident energy of atom, substrate temperature and ion impingement [19]. As the deposition pressure, substrate temperature and pulsed bias all keep constant in this study, the mobility of the adatoms during growth stays the same level and then the grain size can thus remain stable.

\subsection{Chemical Compositions and States}

To further confirm the composition and chemical states of $\mathrm{ZrN}_{x}$ coatings, the XPS spectra are investigated. The adventitious carbon $\mathrm{C} 1 s$ peak with the binding energy of $284.6 \mathrm{eV}$ is utilized to calibrate the binding energies. Figure $5 \mathrm{a}$ is the representative XPS spectra of $\mathrm{ZrN}_{x}$ film showing the photoelectron peaks from $\mathrm{Zr}, \mathrm{N}, \mathrm{O}$ and $\mathrm{C}$ after Ar plasma etching for $0 s, 200 s$ and 500s. It can be seen that the peaks from $\mathrm{O}$ and $\mathrm{C}$ disappear after etching for $200 s$ and the spectrum after etching for $500 s$ is same with the 200 s.

High-resolution spectra are recorded for each individual photoelectron peak, and the atomic concentration is calculated by the relative ratio of the peaks area in the high-resolution XPS spectra, using the standard atomic sensitivity factors of the instrument (N: 0.477, O: 0.78 and $\mathrm{Zr}: 2.576)$. Figure $5 \mathrm{~b}$ is the typical atomic concentration of $\mathrm{ZrN}_{x}$ film (S3) at different etching times. The results agree well with the analysis of the wide scan spectra above. Up to 56.6 at.\% of the oxygen was found in the $\mathrm{ZrN}_{x}$ film without Ar plasma etching and then down to 2.6 at.\% after etching for $200 \mathrm{~s}$ and $500 \mathrm{~s}$. The atomic concentrations of $\mathrm{Zr}$ and $\mathrm{N}$ show the similar tendency with the increase in etching time. They both increase from $0 s$ to $200 s$ and then keep consistent at 500s. This indicates that surface contaminants have been removed significantly after Ar plasma etching for 200s. And the 2.6 at.\% of oxygen may be introduced by the residual oxygen gas in the chamber during deposition.

The atomic concentrations calculated after Ar plasma etching for 500s are selected as the compositions of $\mathrm{ZrN}_{x}$ films. Figure $6 \mathrm{a}$ shows the chemical compositions of the $\mathrm{ZrN}_{x}$ films deposited at different nitrogen flow rates. It can be seen that $\mathrm{Zr}$ content decreases from 55.7 to 49.8 at. $\%, \mathrm{~N}$ content increases from 41.6 to 47.3 at. $\%$, and $\mathrm{O}$ content keeps at about 2.5 at. \% with the increase in nitrogen flow rate. And the nitrogen content $x$ can thus be adjusted over a range from 0.74 to 0.95 , as shown in Fig. 6b. According to the reports and analysis of XRD results above, $V_{\mathrm{N}}$ is believed to be formed in the rocksalt crystal lattice of substoichiometric $\mathrm{ZrN}_{x}$ films. And the $V_{\mathrm{N}}$ concentration can be calculated by comparing the nitrogen content $x$ with the standard stoichiometry ratio. Figure $6 \mathrm{~b}$ shows the variation of $V_{\mathrm{N}}$ concentration versus nitrogen flow rate. It 

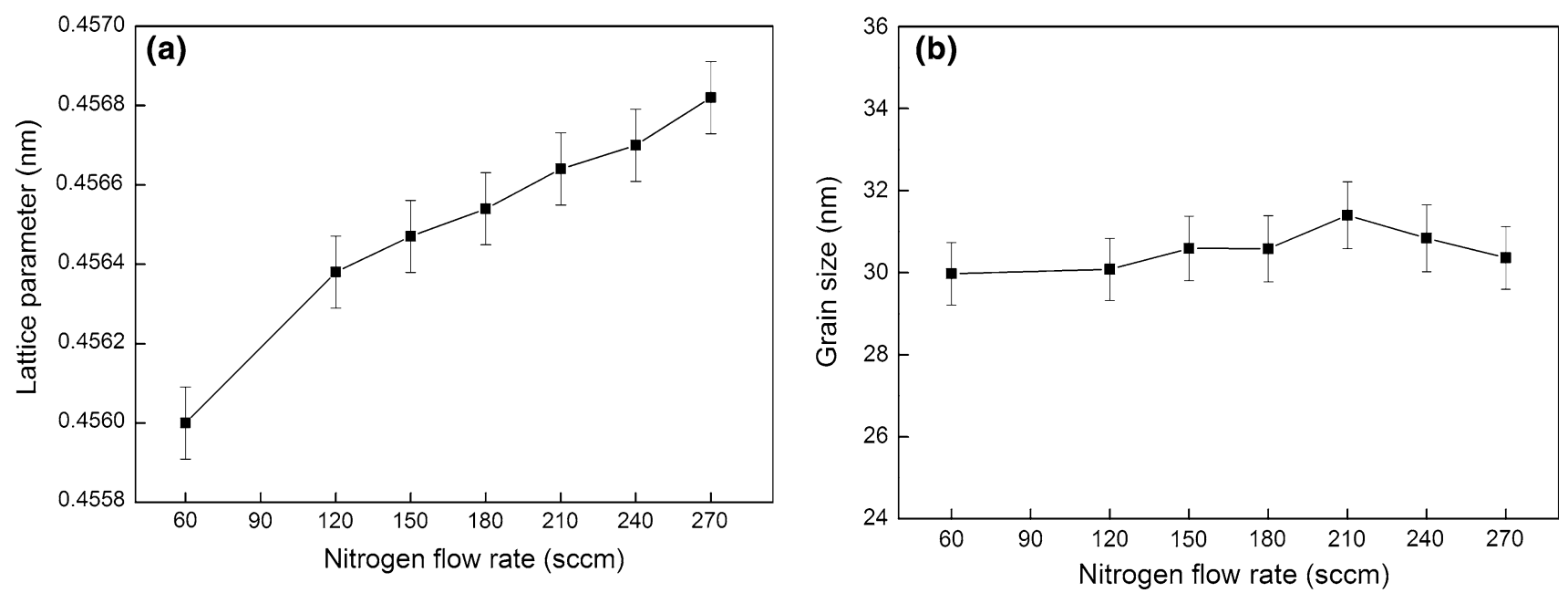

Fig. 4 Lattice parameters $\mathbf{a}$ and grain sizes $\mathbf{b}$ of $\mathrm{ZrN}_{x}$ films deposited at different nitrogen flow rates
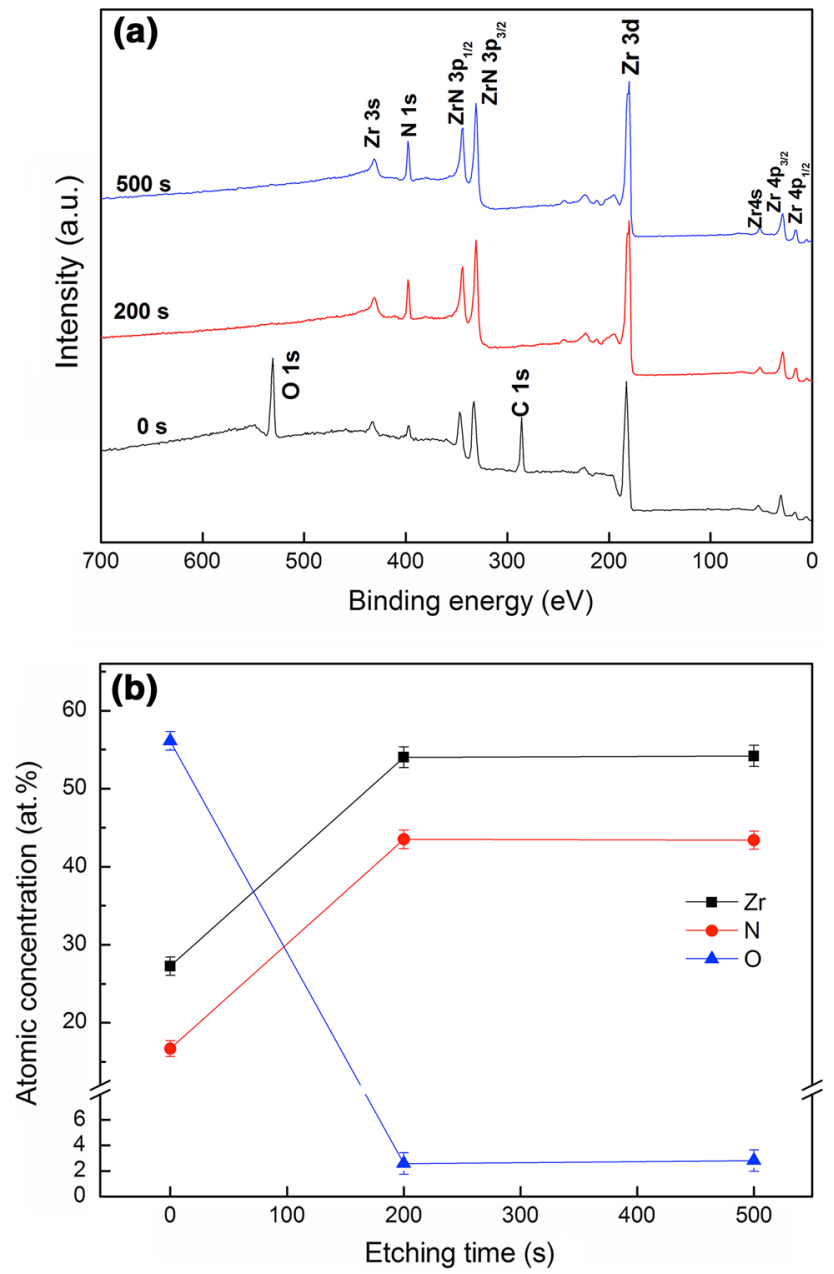

Fig. 5 XPS wide scan spectra a and atomic concentration versus etching time $\mathbf{b}$ of $\mathrm{ZrN}_{x}$ film deposited at a nitrogen flow rate of $120 \mathrm{sccm}$ can be seen that the $V_{\mathrm{N}}$ concentration changes from 26 to $5 \%$ with the increase in nitrogen flow rate.

To confirm the chemical states of $\mathrm{ZrN}_{x}$ films, the deconvolution of high-resolution XPS spectra corresponding to $\mathrm{Zr} 3 d$ and $\mathrm{N} 1 s$ after etching for $500 s$ is studied. Figure 7 shows the representative high-resolution XPS $\mathrm{Zr}$ $3 d$ (a) and $\mathrm{N} 1 s$ (b) spectra of $\mathrm{ZrN}_{x}$ film deposited at 120 sccm. The $\mathrm{Zr} 3 d$ peak is found mainly to consist of individual peaks corresponding to $\mathrm{ZrN}$ : $\mathrm{Zr} 3 d_{5 / 2}$ at $179.9 \mathrm{eV}$ and $\mathrm{Zr} 3 d_{3 / 2}$ at $182.33 \mathrm{eV}$ [4]. The smaller peaks are attributed to $\mathrm{Zr}(\mathrm{O}, \mathrm{N})$ formed by the residual oxygen gas in the chamber during deposition, which can be assigned to $\mathrm{Zr}$ $3 d_{5 / 2}$ at $182.2 \pm 0.2 \mathrm{eV}$ and $\mathrm{Zr} 3 d_{3 / 2}$ at $184.45 \mathrm{eV}$ [4, 20]. High-resolution N $1 s$ spectra are found to be with contribution from nitrogen peaks corresponding to $\mathrm{ZrN}$ at $397.6 \mathrm{eV}$ and $\mathrm{Zr}(\mathrm{O}, \mathrm{N})$ at $399.7 \mathrm{eV}$ [20]. The XRD and XPS results confirm that the substoichiometric $\mathrm{ZrN}_{x}$ films with different $V_{\mathrm{N}}$ concentrations perform the stable rocksalt structure and chemical states.

\subsection{Residual Stress}

Residual stress of the $\mathrm{ZrN}_{x}$ films is obtained by modified XRD $\sin ^{2} \psi$ method [13]. X-ray is incident at an angle of $2^{\circ}$ to increase the diffraction volume of the thin film specimen, and the (220) diffraction is selected to derive the variation of lattice spacing among different $\psi$ angles. With Young's modulus of $460 \mathrm{GPa}$ and Poisson's ratio of 0.186 $[21,22]$, the residual stresses of $\mathrm{ZrN}_{x}$ films are then calculated from the XRD data and shown in Fig. 8. It can be seen that the residual stress remains stable at around $-3.2 \mathrm{GPa}$, showing no significant dependence on the $V_{\mathrm{N}}$ concentration. Because the pulsed bias, deposition 

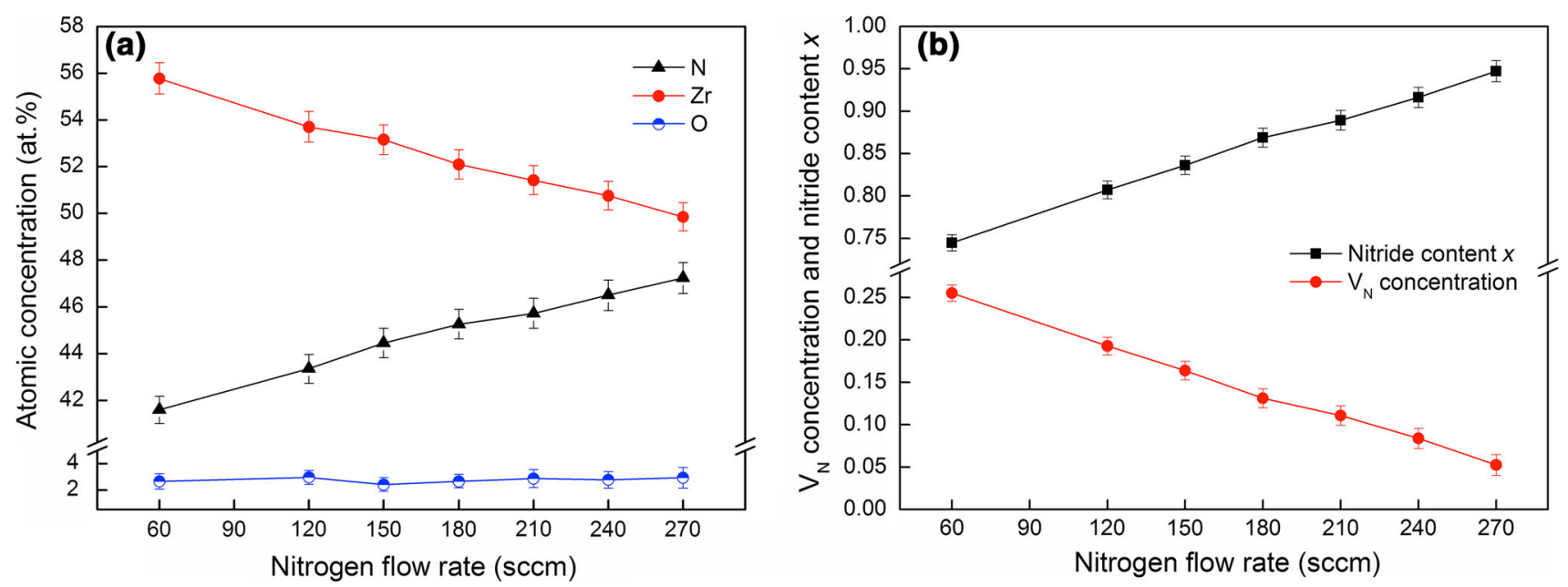

Fig. 6 Atomic concentration a, nitrogen content $x$ and $V_{\mathrm{N}}$ concentration $\mathbf{b}$ of $\mathrm{ZrN}_{x}$ films versus nitrogen flow rate
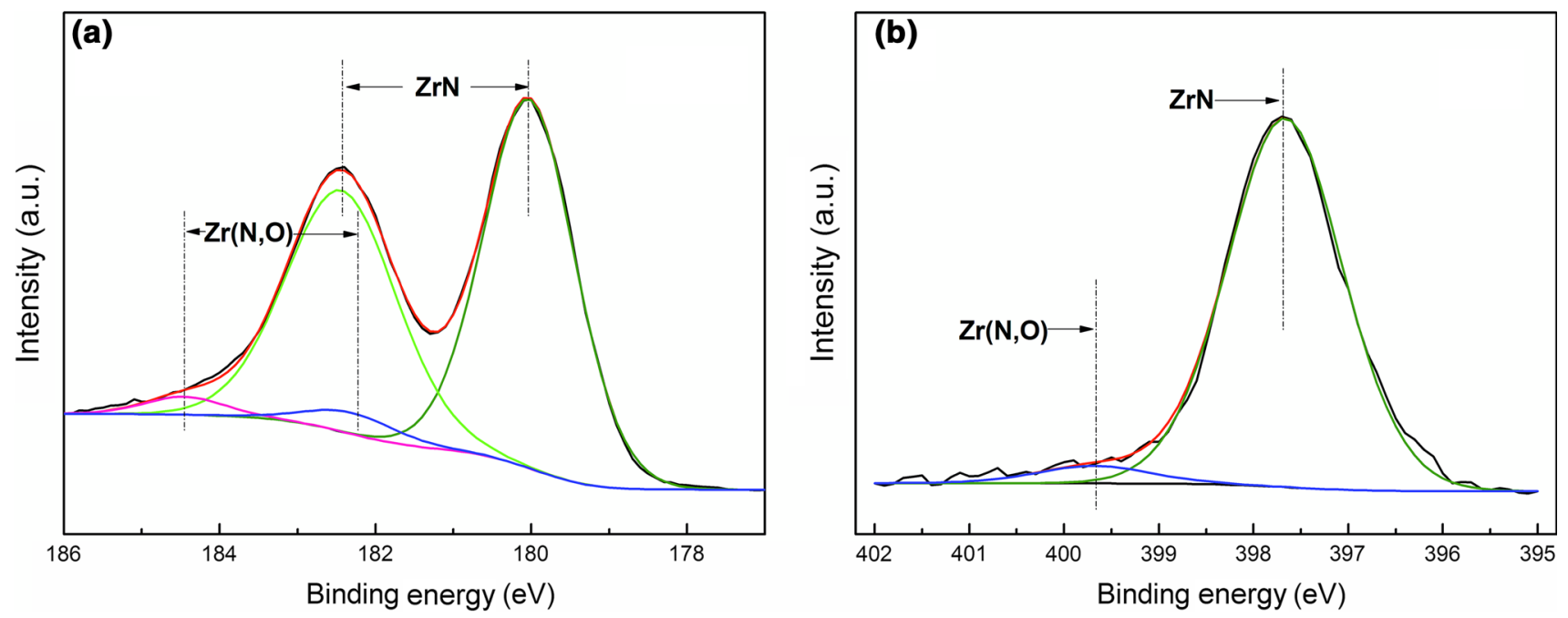

Fig. 7 High-resolution XPS Zr $3 d$ a and N $1 s$ b spectra of $\mathrm{ZrN}_{x}$ film deposited at a nitrogen flow rate of $120 \mathrm{sccm}$

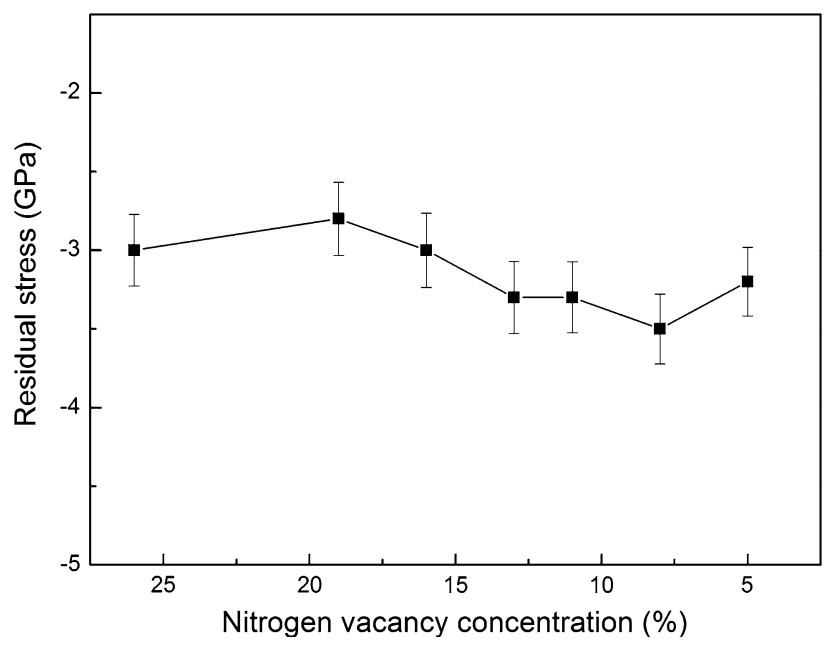

Fig. 8 Residual stress of $\mathrm{ZrN}_{x}$ films versus $V_{\mathrm{N}}$ concentration temperature and working pressure are constant, the plasma sheath above the substrates keeps stable. The energy of nitrogen and zirconium ions can thus keep constant at different nitrogen flow rates. So the same growth state and deposition rate of $\mathrm{ZrN}_{x}$ films are observed. And also because $\mathrm{ZrN}_{x}$ has the wide solid solution region, the films with different nitrogen content $x$ all perform the stable rocksalt structure and lattice distortion in this work. So the residual stress can thus keep constant.

\subsection{Hardness and Elastic Modulus}

The nanohardness and elastic modulus of $\mathrm{ZrN}_{x}$ film versus $V_{\mathrm{N}}$ concentration are demonstrated in Fig. 9. The hardness first increases from $27.6 \mathrm{GPa}$ at $26 \%$ to the maximum of $29.6 \mathrm{GPa}$ at $16 \%$ and then gradually decreases to $26.8 \mathrm{GPa}$ at $5 \%$ with decrease in $V_{\mathrm{N}}$ concentration. The elastic 


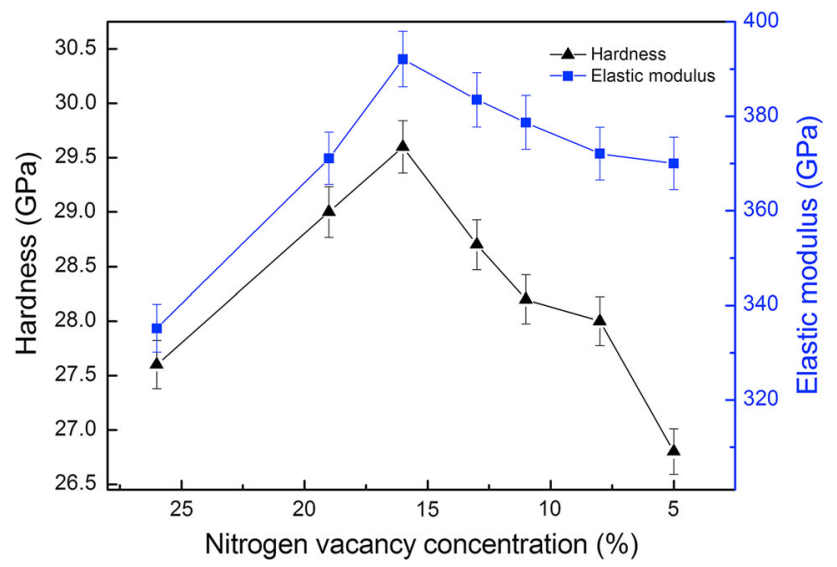

Fig. 9 Hardness and elastic modulus of $\mathrm{ZrN}_{x}$ films versus $V_{\mathrm{N}}$ concentration

modulus shows the similar variation tendency with the hardness, reaching the top of $392.1 \mathrm{GPa}$ at $V_{\mathrm{N}}$ concentration of $16 \%$. The variation tendency is similar with many studies of other researchers and also our previous work about $\operatorname{TiN}_{x}[9,10,23]$, while the reasons for higher hardness compared to 25.0 GPa reported by Singh et al. [9] and 26.5 GPa by Huang et al. [11] may be from the excellent deposition quality and strong preferential growth along (111) orientation in this work.

It is well known that several conventional meso-scale factors may contribute to the material strength of hard thin films marked with hardness and elastic modulus, such as crystallographic texture, grain size, roughness and the lattice distortion (residual stress) [24-26]. In this study, the test results show that all the $\mathrm{ZrN}_{x}$ films have excellent deposition qualities and similar morphologies. And within the large $V_{\mathrm{N}}$ concentration range (26-5\%), $\mathrm{ZrN}_{x}$ films perform the stable rocksalt single-phase structure and keep the preferred orientation unchanged. In addition, the film thicknesses, grain sizes and residual stresses are also almost constant. Therefore, it is confirmed that the influences of conventional meso-scale factors on the hardness variation have been significantly controlled and the $V_{\mathrm{N}}$ concentration-dependent peak values may be originated only from the atomic-scale factors, such as different energy band states and electronic structures caused by the change in $V_{\mathrm{N}}$ concentration. This is also the reason why the hardness variation is not so large.

The theory simulation about atomic-scale strengthening mechanism of transition-metal carbonitrides $\mathrm{TMC}_{x} \mathrm{~N}_{1-x}$ has been reported by Jhi et al. [27], which may be an important reference to how to understand and explain the phenomenon in this work. It was reported that the highly directional coupling between transition-metal electrons and non-metal electrons can result in a strongly shear resistive covalent $p d \sigma$ energy band and such band will give a positive contribution to the hardness, while the metallic $\sigma$ band state derived from $d-d$ interactions is unstable against shear deformations and will give a negative contribution. In our work, more valence electrons are brought in with the decrease in $V_{\mathrm{N}}$ concentration and most of them gradually fill the covalent $p d \sigma$ band formed by $\mathrm{Zr} 4 d$ and $\mathrm{N} 2 p$ states and few fill the metallic $\sigma$ band formed by $\mathrm{Zr} 4 d$ states at first, which give a rise to the hardness and elastic modulus. And the following decrease in hardness and elastic modulus is attributed in large part to the rapidly filling into metallic $\sigma$ band after the covalent $p d \sigma$ band was completely filled. The greatest hardness of transition-metal carbonitrides $\mathrm{TMC}_{x} \mathrm{~N}_{1-x}$ reported by Jhi et al. [27] was expected at the stoichiometry point which gives a valence electron concentration (VEC) of 8.40 per cell. In this work, the best performances of the $\mathrm{ZrN}_{x}$ films are obtained at $V_{\mathrm{N}}$ concentration of $16 \%$. Considering the contribution of residual oxygen, the VEC of $\mathrm{ZrN}_{x}$ film at $V_{\mathrm{N}}$ concentration of $16 \%$ is 8.48 as shown in Fig. 10 (outermost electron configuration of $\mathrm{Zr}, \mathrm{N}$ and $\mathrm{O}$ are $4 d^{2} 5 s^{2}, 2 s^{2} 2 p^{3}$ and $2 s^{2} 2 p^{4}$ ), which agrees well with the calculated value of 8.40 reported by Jhi et al.

\subsection{Electrical Properties}

Electric conductivity $\left(\sigma_{\mathrm{e}}\right)$, carrier concentration $(n)$ and mobility $(\mu)$ of the $\mathrm{ZrN}_{x}$ films with different $V_{\mathrm{N}}$ concentrations are investigated by Hall measurements system using the van der Pauw configuration under $0.558 \mathrm{~T}$ magnetic field at room temperature. The dependence of conductivity on $V_{\mathrm{N}}$ concentration is illustrated in Fig. 11. It can be observed that the conductivity is sensitive to the variation of $V_{\mathrm{N}}$ concentration. It keeps stable around $9.0 \times 10^{3} \mathrm{~S} / \mathrm{cm}$ at first and then increases to $3.7 \times 10^{4} \mathrm{~S} / \mathrm{cm}$ suddenly around $V_{\mathrm{N}}$ concentration of $15 \%$ and decreases gradually to $1.5 \times 10^{4} \mathrm{~S} / \mathrm{cm}$ finally.

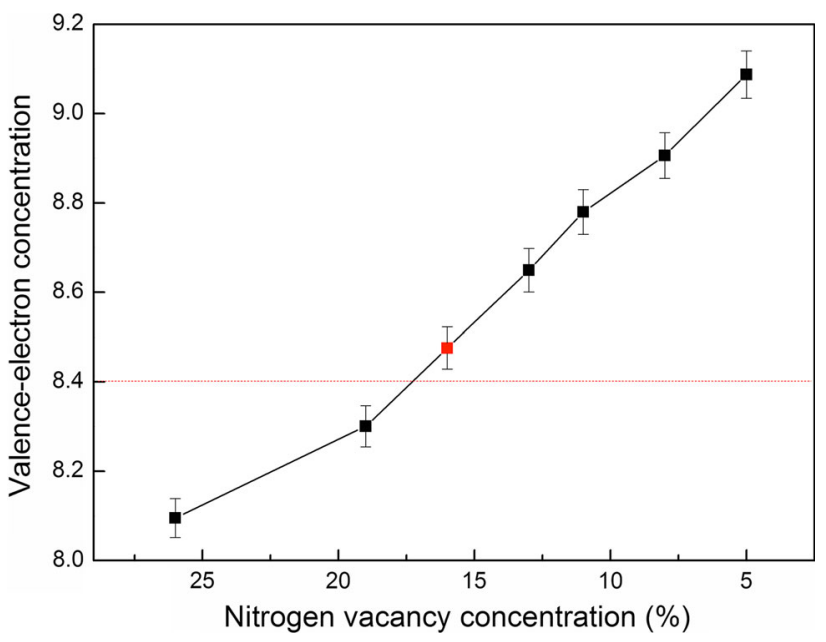

Fig. 10 Valence-electron concentration of $\mathrm{ZrN}_{x}$ films versus $V_{\mathrm{N}}$ concentration 


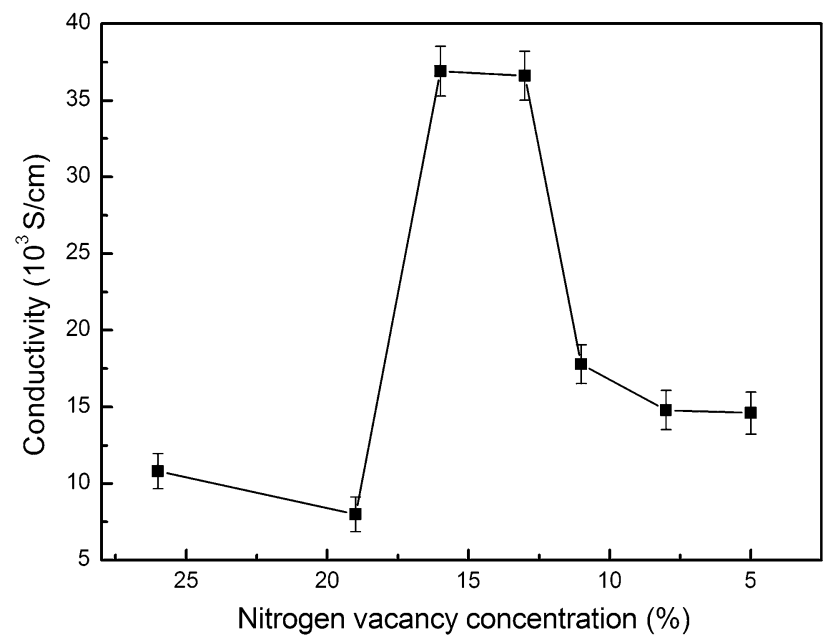

Fig. 11 Conductivity of $\mathrm{ZrN}_{x}$ films versus $V_{\mathrm{N}}$ concentration

The conductivity is a little higher than that of the standard nitride semiconductor materials such as gallium nitride and indium nitride [28], but the $V_{\mathrm{N}}$ formed in crystal lattice could act as the donor-like defect which makes the behavior of electrical properties of substoichiometric $\mathrm{ZrN}_{x}$ films be very similar to the semiconductor $[29,30]$. So the electric conductivity of $\mathrm{ZrN}_{x}$ films can be regarded as a product of carrier concentration and mobility by the formula: $\sigma_{\mathrm{e}}=n q \mu$ ( $q$ is charge quantity of carrier) [31]. Figure 12 shows the carrier concentration and mobility of $\mathrm{ZrN}_{x}$ films versus $V_{\mathrm{N}}$ concentration. The carrier concentration shows a similar tendency with conductivity while the mobility changes in an opposite way. So the carrier concentration is perhaps the most critical variable in determining the tendency of electric conductivity. With respect to the analysis above, more valence electrons are brought in with the decrease in $V_{\mathrm{N}}$ concentration and most of them gradually fill the covalent $p d \sigma$ band and few fill the metallic $\sigma$ band before VEC of 8.40.

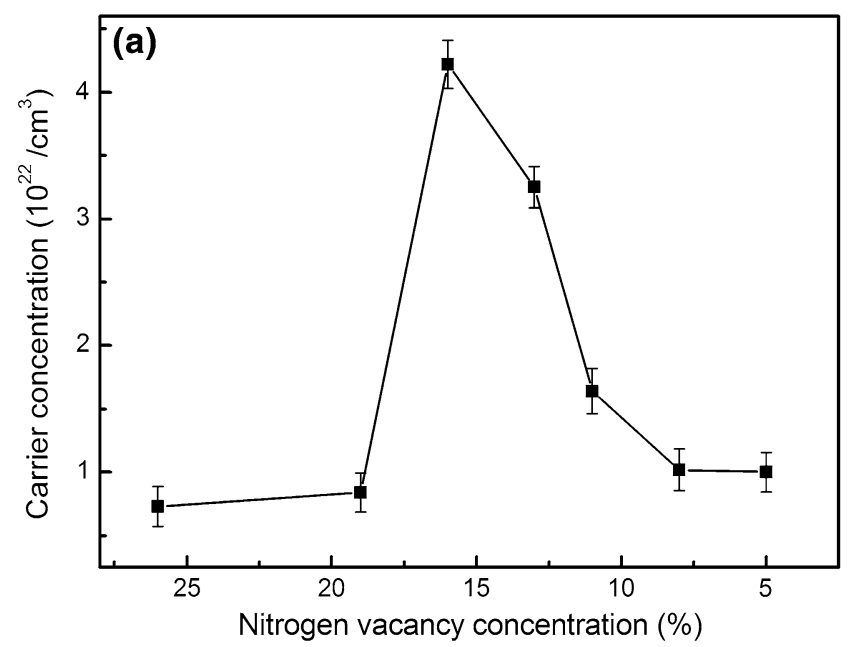

The covalent $p d \sigma$ band is below the Fermi level which contributes little to the conduction electron, and the metallic $\sigma$ band is above the Fermi level which can increase the conduction electron $[32,33]$. So before VEC of 8.40 , the valence electrons brought by the filling of $V_{\mathrm{N}}$ contribute little to the carrier concentration and thus it keeps stable, while the sudden increase in carrier concentration at VEC of 8.48 can be ascribed to the rapid filling of metallic $\sigma$ band after the covalent $p d \sigma$ band is completely filled at VEC of 8.40. And the reason for the following gradual decrease in carrier concentration may come from the negative contribution of the vanish of $V_{\mathrm{N}}$. The $V_{\mathrm{N}}$ could act as donor-like defects and add extra free electrons to the conduction band [30]. As the $V_{\mathrm{N}}$ is filled gradually with the increase in nitrogen content, the negative contribution to the carrier concentration becomes more apparent, so the decreasing tendency is observed.

The carrier mobility of $\mathrm{ZrN}_{x}$ films versus $V_{\mathrm{N}}$ concentration shows an opposite way with carrier concentration. This tendency can be regarded as the combined effect of electron scattering due to the carrier and $V_{\mathrm{N}}$ defects. With the increase in carrier concentration, the electron scattering due to the carrier will be heightened and so the mobility will decrease. This is the major factor that determines the opposite tendency with carrier concentration. And the gradual vanish of $V_{\mathrm{N}}$ will accelerate the weakening of the electron scattering of $V_{\mathrm{N}}$ defects, which also contribute to the tendency of mobility.

\section{Conclusion}

High-quality $\mathrm{ZrN}_{x}$ films with different $V_{\mathrm{N}}$ concentrations were successfully synthesized on the $\mathrm{Si}$ (111) substrates by varying the nitrogen flow rate using the method of

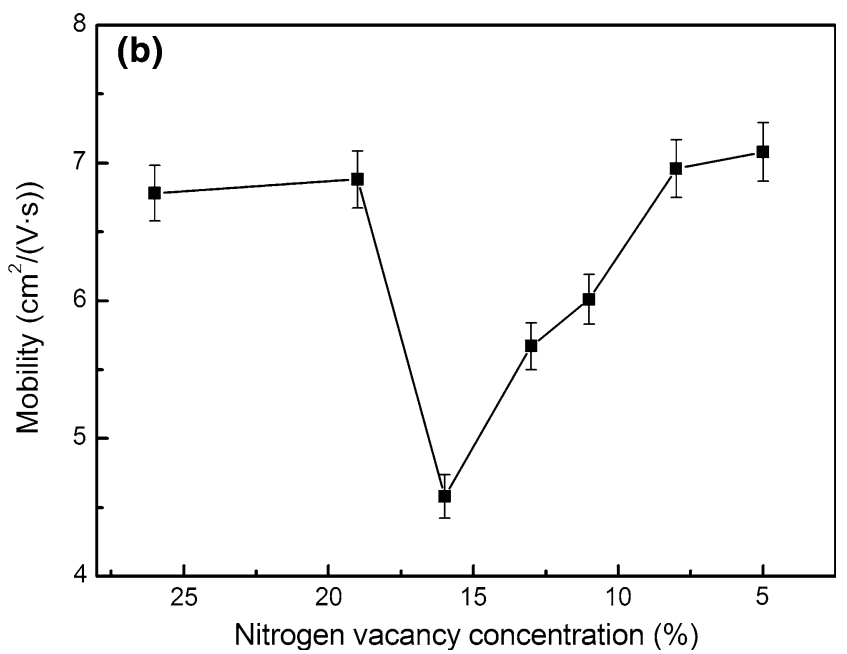

Fig. 12 Carrier concentration a and mobility $\mathbf{b}$ of $\mathrm{ZrN}_{x}$ films versus $V_{\mathrm{N}}$ concentration 
enhanced magnetic filtering arc ion plating. The $\mathrm{ZrN}_{x}$ films all exhibited stable rocksalt single-phase structure and kept the preferred orientation unchanged within the large $V_{\mathrm{N}}$ concentration range (26-5\%). The thickness, grain size and residual stress of $\mathrm{ZrN}_{x}$ films with different $V_{\mathrm{N}}$ concentrations were not changed so much. Both the nanohardness and elastic modulus of $\mathrm{ZrN}_{x}$ films first increased and then decreased with the decrease in $V_{\mathrm{N}}$ concentration, and the maximums were obtained at 16\%: $29.6 \mathrm{GPa}$ and $392.1 \mathrm{GPa}$. The electric conductivity was also found highly dependent on the $V_{\mathrm{N}}$ concentration and showed a similar tendency with nanohardness. The $V_{\mathrm{N}}$ concentration-dependent mechanical and electrical enhancement of the $\mathrm{ZrN}_{x}$ films in this work was found from the different atomic-scale electronic band structure, rather than the conventional meso-scale factors, such as crystallographic texture, grain size and residual stress.

Acknowledgements This work was supported financially by the National Natural Science Foundation of China (No. 51271047).

\section{References}

[1] L. Toth, Transition Metal Carbides and Nitrides (Academic Press, New York, 1971)

[2] R. Yu, Y.H. Jiang, J. Feng, R.F. Zhou, Y.Q. Zhang, R. Zhou, J. Mater. Sci. 3443, 48 (2013)

[3] L. Hultman, Vacuum 1, 57 (1999)

[4] I. Milošev, H.H. Strehblow, B. Navinšek, Thin Solid Films 246, 303 (1997)

[5] W.J. Chou, G.P. Yu, J.H. Huang, Surf. Coat. Technol. 59, 167 (2003)

[6] C.J. Xuan, Z. Zhao, P.G. Jönsson, J. Eur. Ceram. Soc. 2435, 36 (2016)

[7] L. Tsetseris, N. Kalfagiannis, S. Logothetidis, S.T. Pantelides, Phys. Rev. Lett. 99, 125503 (2007)
[8] N.J. Ashley, R.W. Grimes, K.J. McClellan, J. Mater. Sci. 1884, 42 (2007)

[9] A. Singh, P. Kuppusami, S. Khan, Appl. Surf. Sci. 280, 117 (2013)

[10] B. Abdallah, M. Naddaf, M.A. Kharroub, Nucl. Instrum. Methods B 298, 55 (2013)

[11] J.H. Huang, C.H. Ho, G.P. Yu, Mater. Chem. Phys. 102, 31 (2007)

[12] S. Khan, M. Mehmood, I. Ahmad, Mater. Sci. Semicond. Process. 486, 30 (2015)

[13] C.H. Ma, J.H. Huang, H. Chen, Thin Solid Films 73, 418 (2002)

[14] M.D. Huang, G.Q. Lin, Y.H. Zhao, Surf. Coat. Technol. 109, $176(2003)$

[15] Y.H. Zhao, W.J. Yang, C.Q. Guo, Acta Metall. Sin. (Engl. Lett.) 984, 28 (2015)

[16] G.Q. Lin, Y.H. Zhao, H.M. Guo, J. Vac. Sci. Technol. A 1218 , 22 (2004)

[17] P.E. Schmid, S.M. Sato, F. Lévy, J. Vac. Sci. Technol. A 2870, 16 (1998)

[18] G.F. Duan, G.L. Zhao, L. Wu, Appl. Surf. Sci. 2428, 257 (2011)

[19] K.W. Whang, Y.W. Seo, J. Vac. Sci. Technol. A 1496, 11 (1993)

[20] D. Roman, J. Bernardi, C.L.G.D. Amorim, Mater. Chem. Phys, 147, 130 (2011)

[21] E. Torok, A.J. Perry, L. Chollet, Thin Solid Films 37, 153 (1987)

[22] A.J. Perry, Thin Solid Films 463, 193-194 (1990)

[23] K.C. Han, G.Q. Lin, C. Dong, Ceram. Int. 10332, 42 (2016)

[24] W.J. Chou, G.P. Yu, J.H. Huang, Surf. Coat. Technol. 206, 140 (2001)

[25] H. Jiménez, E. Restrepo, A. Devia, Surf. Coat. Technol. 1594, 201 (2006)

[26] Z.B. Qi, P. Sun, F.P. Zhu, Surf. Coat. Technol. 3692, 205 (2011)

[27] S.H. Jhi, J. Ihm, S.G. Louie, Nature 132, 399 (1999)

[28] I. Vurgaftman, J.R. Meyer, J. Appl. Phys. 3675, 94 (2003)

[29] X.C. Wang, X.M. Chen, B.H. Yang, Solid State Sci. 435, 14 (2012)

[30] Z.Q. Gu, C.Q. Hu, X.F. Fan, Acta Mater. 315, 81 (2014)

[31] D.A. Neamen, An Introduction to Semiconductor Devices (McGraw-Hill Press, New York, 2006)

[32] B. Saha, J. Acharya, T.D. Sands, J. Appl. Phys. 33715, 107 (2010)

[33] C. Stampfl, W. Mannstadt, R. Asahi, Phys. Rev. B 155106, 63 (2001) 